\title{
Professional Socialization in Nursing; Experiences of New and Established Nursing Staff in Response to Pa- tient Death
}

\author{
Janet Wilson* \\ Senior Lecturer (Nursing), Faculty of Health and Wellbeing, Sheffield Hallam University, Sheffield \\ *j.l.wilson@shu.ac.uk
}

\begin{abstract}
This paper describes one component; professional socialisation, from the findings of a larger study exploring the responses of Registered Nurses and healthcare support workers to patient death in an acute hospital setting. Professional socialization involves the acquisition of knowledge and skills of a particular profession, and the adoption of values and norms leading to the development of behaviours and self-concept that are indicative of the characteristics necessary for the role taken at work. It is recognised that there is a strong desire for nurses to feel part of a team and as a result conform to existing practices and routines in order to be accepted.
\end{abstract}

A Heiddegarian phenomenological approach was used to gather and analyse data through individual interviews. Participants were recruited through purposive sampling from two medical wards in a large teaching hospital in the United Kingdom.

It was found that recently qualified Registered Nurses felt they should not show their emotions or discuss their feelings with certain colleagues. In order to be accepted by established staff on the ward they felt they needed to conform to behaviours they believed would be acceptable. Some of the more experienced Registered Nurses and healthcare support workers showed their emotions and talked about events to colleagues expressing the benefits they perceived of doing this for both themselves and others. This demonstrates that newly qualified staff were not always correct in their assumptions of what was considered the norm in these situations.

Some of the newer Registered Nurses felt they needed to be more rigid in their behaviours, and were reluctant to challenge medical staff regarding active treatments. These differences could be due to established Registered Nurses feeling secure and comfortable in their professional roles, and being clear on the values they hold. More inexperienced Registered Nurses could be lacking in both knowledge and skills, as well as being unsure of their professional role.

Keywords: Professional Socialisation, Belongingness, Self-Image, Nursing, Sense of Belonging, Professional Identity

\section{INTRODUCTION}

Professional socialisation can be defined as the acquisition of values, behaviours and attitudes necessary to assume a professional role (Shinyashiki et al 2006). The process of socialisation is one that occurs in all cultures and groups. Berger and Luckman (1967) distinguished between primary socialisation, which occurs in childhood within the family, and secondary socialisation occurring when adults become socialised into wider society. Part of this involves being socialised into a profession or work setting. Cohen (1981) defined professional socialisation as being a complex process involving a person acquiring the knowledge and skills of a particular profession, along with a sense of occupational identity characteristic of that profession. This involves adopting values and norms, which then become internalised, affecting behaviours and the individual's self-concept. 
Feng and Tsai (2012) identified that feeling a sense of belonging was very important to newly qualified nurses and that in order to feel part of the team they felt they had to conform to the norms for that particular ward. Conflicts they encountered between the professional values they were taught as students and the organisational values they experienced in the clinical setting had to be managed. Levett-Jones and Lathlean (2008) studied the concept of belongingness, specifically with nursing students in clinical areas. Their findings were that students quickly learnt that in order to feel that they belonged, and were accepted by other staff, they adopted the behaviours and practices they saw on the wards, even if these were recognised as not being the best evidence based practice. This research agrees with the findings of similar studies. Champion et al (1998) found that new staff adopted existing values and norms in clinical areas, adapting their behaviour in order to be accepted. McKenna et al (2003) found that new staff complied with established ward practices and routines in order to be accepted and, if any resisted aspects of the ward culture, there was conflict resulting in emotional and physical distress.

Mooney (2007) identified two features of the socialisation process when she studied how professional socialisation was the key to survival for newly qualified nurses. The first was the acceptance of the ritualistic practices and routines, which took precedence over other aspects such as psychological care. In order to be highly thought of by existing staff and to fit in with the ward routine, the newly qualified staff conformed to these practices. The second feature involved the new staff feeling vulnerable and powerless. They were referred to as 'the juniors' emphasising the importance of hierarchy and the supremacy of other staff, and found that any errors they made were highlighted disproportionately to those of other staff. As junior staff, they also had limited choices regarding the shifts they worked and holiday choices. It has been suggested that obedience and conformity are part of the nursing socialisation process and that this has led to a lack of autonomy and assertiveness to question practices in nursing (Parsons and Griffiths 2006). McKenna et al (2003) found in their study of newly qualified nurses that those who questioned existing practices could be subject to verbal abuse, humiliation and exclusion by their colleagues in order to intimidate them into conforming to accepted norms. Parsons and Griffiths (2006) found in a study of midwives, that two thirds of those surveyed conformed without question to their hospitals' routine. An example cited concerned oral intake during labour, where mothers in labour were refused food even though there was no evidence to support this practice.

Mackintosh (2006) highlights that these negative aspects of professional socialisation can possibly lead to the maintenance of ritualised practice and traditional views, resulting in the loss of idealism and innovation.

Another aspect of socialisation is the adoption of roles, experiments have demonstrated that people who are given certain roles act out the behaviour they think is expected of them in that role. This includes what are considered norms in that setting, and these behaviours quickly become genuine for them (Myers 2012). An example of this is the experiment by Zimbardo (1971) of giving the role of prison warders and prisoners to a group of college students. This study had to be stopped prematurely as the students playing warders devised cruel and degrading routines for their fellow student prisoners, demonstrating that far from being powerless to resist imposed roles, what is initially 'unreal' can evolve into what is 'real'.

In a hospital ward, staff caring for those who die can take on a role of responding in a certain way, which at first may be false but quickly becomes genuine. An explanation offered for this is self-presentation theory (Leary 2004). In order to make a good impression and appear competent at work, certain responses and behaviours are acted out. Initially, this may be done by copying the way they see colleagues behaving, to fit in with the norms they see around them. However these behaviours can then become genuine.

\section{RESEARCH STUDY}

Professional socialisation was an aspect identified from a wider study exploring the responses of nursing staff to patient death in an acute medical ward in a large teaching hospital in the United Kingdom (UK).

Volume 2016

Page 2 
The objectives of the study included identifying the responses of registered nurses in terms of thoughts and behaviours, and discovering factors staff perceived as influencing their responses.

\section{METHOdOLOGY AND DATA ANALYSIS}

Heideggarian phenomenology was the methodology used in this study as the aim was to explore the lived experiences of the participants in relation to their responses when patients they had cared for died. The participants who were recruited through purposive sampling were eight Registered Nurses (RN) and five healthcare support workers (HSW). Selection criteria were that participants had to have experience of caring for patients in this setting who had died. Personal accounts were obtained through unstructured and semi structured individual interviews. Ethical and research governance approval was sought and granted by the local National Health Service (NHS) and university committees and each participant signed forms giving their informed consent to be part of the study.

The researcher recorded and transcribed the interviews and repeated thematic analysis was used to identify themes.

\section{Findings}

The two themes relevant to this issue were: beliefs about what behaviour was viewed as acceptable from other nursing staff on the ward, and strategies participants used to manage and modify their emotional responses in order to conform to what they perceived to be acceptable.

$>$ Three types of perceived expectations were identified,

Organisational; concerned with the running of the ward and workload

$>$ Personal; concerned with the individual emotional responses of staff

Professional; participants views on how a 'professional nurse' is expected to respond in this situation.

\section{Organisational}

RNs identified the pressures they felt to get on with their other nursing duties and to meet the constant demand for vacant beds.

"Its very rushed, we need to get the patient to the mortuary quickly. The bed manager's asking for the bed as we need it for somebody else" (RN5)

"You've got to get on with the next job; admitting someone, doing the drugs or whatever" (RN1)

\section{Personal}

Both RNs and HSWs reported experiences of being told how they should behave in response to a patient's death.

"If I get upset, my colleague says 'You've got to leave it at work',

and I try to do that, but sometimes it's difficult" (HSW12)

"I think we hear that expression a lot as Nurses:

Put your feelings in a box and move on" (RN10)

"I knew she [a new HSW] was going to cry and I said "don't you dare" (HSW7)

\section{Professional}

As well as overt instructions given to staff, many RNs felt there were expectations which were implicit as part of the culture of the ward directing how they should act as a healthcare professional in response to a patient's death. 


\section{American Research Journal of Nursing (ARJN)}

"I think there's a big expectation that you can cope, because

It's an expected part of your role" (RN10)

"The emphasis for senior staff is to support more junior colleagues

which is what I do, but sometimes you need a bit of support yourself" (RN6)

Although the length of time staff had worked in this area was not part of the selection criteria for participants, the findings identified differences amongst staff who had been working in the area for less than two years and those whom were experienced some of which had worked in this setting for over ten years.

In order to appear professional, competent and accepted RNs who had qualified within the last two years reported that they felt they needed to demonstrate a range of behaviours in their work. These were; to appear efficient by carrying on with their work after a traumatic event such as patient death, complete tasks within time constraints they often imposed on themselves, not show emotions of being upset and not talking about the death and how this affected them to certain colleagues.

They considered these features to be existing practices on the ward and in order to be accepted they needed to conform to these behaviours.

In contrast some of the more experienced RNs described how they had cried with relatives

"I Know some people think it is wrong, but i have cried with

Relatives. I think $t$ shows that we're human and that we care.

I think sometimes we try and put a brave face

On things and we come across as being quite heartless" (rn5)

They also reported talking to colleagues about specific deaths

"We text one another and ring one another [colleagues] and

Have a good natter... get it off our chests and its brilliant" (rn6)

Some experienced nurses also talked about death to other patients on the ward in certain situations.

"Now, after this many years,(in nursing) i do tell the other patients,

They know and $i$ think it just helps them to say 'john's died'.

"It's like you pull the curtains round when the mortuary trolley comes

And go through all these euphemisms for the mortuary, i don't do

It anymore, $i$ use the proper words". (rn1)

\section{DISCUSSION}

The findings from this research indicate that staff who were new to this area of work were not always correct in their assumptions of what they considered experienced staff would find acceptable in terms of behavioural responses to patient death. Some of the newer RNs felt they needed to be rigid in their behaviours, saying it was never right to cry in front of relatives, and they were reluctant to challenge medical staff regarding active treatments. In contrast some of the experienced nurses freely talked about crying with relatives in certain situations and how they felt this was both acceptable and could be helpful for relatives in showing they were human and cared for the person who died. Some experienced RNs related that they had challenged medical staff about continuing active treatment for patients who were dying, asserting that end of life care was appropriate at this time. These differences could be due to the experienced RNs feeling secure and comfortable in their 
professional roles, having a higher level of knowledge and skills in this area of nursing and being clear on the values they hold. More inexperienced RNs could be lacking in both knowledge and skills, as well as being unsure of their professional role.

Another aspect was talking about the death of patients to colleagues and to other patients. The use of euphemisms was more common in newer staff members whereas the experienced staff talked much more openly about patients having died. These staff also felt more comfortable talking to patients about the death of a fellow patient they knew and giving patients the opportunity to express their sadness at the death. Some newer staff felt they should not talk to patients about the death of another patient stating they would be breaking confidentiality so they just drew curtains round a bed when the mortuary staff arrived and then drew back the curtains to reveal an empty bed without saying anything to patients in neighbouring beds. More experienced staff commented that this led to a conspiracy of silence as other patients knew what had happened but felt they could not say anything creating an awkward atmosphere on the ward. Experienced staff contended the view that if the death was acknowledged then patients could express their sympathy and also have the opportunity to discuss their own anxieties with staff.

The experienced staff also reported creating their own networks of colleagues with whom they could debrief about the death that had occurred and that this was an informal arrangement amongst peers they felt comfortable with. Some newer staff members thought it was not acceptable to talk about how they had been affected by a death but also related how they sometimes lay awake at night thinking about what had happened and had difficulty detaching their thoughts from the event. By talking about the death soon after the event with colleagues this could help staff to debrief and not to have recurrent thoughts about what had happened when they were at home.

Some participants in this study talked about innovative changes they would like to see in regard to more openness in talking about death. This included acknowledgement and discussions with patients who were near the end of their lives and systems in place for staff enabling them to share their experiences. This demonstrates that staff can have new ideas to change and develop practices on the ward, though only a few mentioned taking action to promote these changes. This finding supports the view by Mackintosh (2006) that staff can be reluctant to promote innovative changes as they think they need to maintain traditional practices in order to be accepted by their more experienced colleagues. The promotion of openness amongst staff through opportunities for them to discuss their responses to patient death and how they manage it both professionally and personally could help develop better team working and also enable staff to support each other in this emotional aspect of their work.

\section{CONCLUSION}

A sense of belonging is recognised as being an important issue for nursing staff, particularly those who are recently qualified or new to this work. In order to feel accepted by colleagues, staff often follow the practices of more established members of staff and do this even if it means going against what they know to be the best evidence based practices. The findings from this study demonstrate that newer members of staff can make some incorrect assumptions concerning what experienced staff consider to be acceptable behaviours in response to patient death. For newer staff this could result in them not developing constructive support systems with colleagues and concealing their emotional responses which could affect them outside of the work setting in terms of their psychological health. Several were not aware that more experienced staff actually behaved very differently to the beliefs they had about them in terms of expressing their emotions and talking to colleagues and patients about death. Opportunities for newer staff members to discuss expectations and practices with their more experienced colleagues could help them develop constructive strategies for managing their emotions around patient death and more openness in talking about this issue could lead to improved staff relationships and more effective teamworking. 


\section{REFERENCES}

1. Berger P., Luckman T. (1967) The Social Construction of Reality. London, Penguin Press

2. Champion B., Ambler N. and Keating D. (1998) 'Fitting in; becoming an insider: A nursing perspective.' Nurses Registration Board of New South Wales Report. Newcastle, Australia.

3. Cohen H. (1981) 'The nurse's quest for a professional identity'. In Hawkins E. and Ewans A. (eds\} 'How students experience professional socialisation'. International Journal of Nursing Studies 36(1): 41-49.

4. Feng R. and Tsai Y. (2012) 'Socialisation of new graduate nurses to practising nurses.' Journal of Clinical Nursing 21: 2064-2071.

5. Leary M. (2004) 'The self we know and the self we show: self-esteem, self-presentation and the maintenance of interpersonal relationships.' In Brewer M. and Hewstone M. (eds.) Emotion and Motivation. Malden MA., Usisters.

6. Levett-Jones T. and Lathlean J. (2008) 'Belongingness: A prerequisite for nursing students' clinical practice.' Nurse Education in Practice 8: 103-111.

7. Mackintosh C. (2006) 'Caring: The socialisation of pre-registration student nurses: A longitudinal qualitative descriptive study' International Journal of Nursing Studies 43: 953-962.

8. McKenna B., Smith N., Poole S. and Coverdale J. (2003) 'Horizontal violence: experiences of Registered Nurses in their first year of practice.' Journal of Advanced Nursing 42(1): 90-96.

9. Mooney M. (2007) 'Professional socialisation: The key to survival as a newly qualified nurse'. International Journal of Nursing Practice 13: 75-80.

10. Myers D. (2012) Social psychology (eleventh edition). London, Mcgraw-Hill.

11. Parsons M. and Griffiths R. (2006) 'The effect of professional socialisation on midwives' practice.' Women and Birth 20: 31-34.

12. Shinyashiki G., Mendes I., Trevizan M., Day R., (2006) Professional Socialisation: students becoming nurses. Revista Latino-Americano de Enfermagem 14(4) 601-607

13. Zimbardo P. (1971) 'The psychological power and pathology of imprisonment', in Myers D., Abell J., Kolstad A. and Sani F. Social Psychology (European edition). Maidenhead, McGraw-Hill.

Citation: Janet Wilson, Professional Socialization in Nursing; Experiences of New and Established Nursing Staff in Response to Patient Death. ARJN Volume 2016; pp:1-6

Copyright (C) 2016 Janet Wilson. This is an open access article distributed under the Creative Commons Attribution License, which permits unrestricted use, distribution, and reproduction in any medium, provided the original work is properly cited. 